PRODUCTION

ENGINEERING

ARCHIVES
2015, Vol. 8, No. 3, pp 28-31

ISSN 2353-5156 (print version)

ISSN 2353-7779 (online version)

\title{
Rapid prototyping in medical sciences
}

\author{
Ákos Márk Horváth ${ }^{1}$, Péter Ficzere ${ }^{1}$ \\ ${ }^{1}$ Dept. of Vehicle Elements Vehicle-Structure Analysis, 1111 Budapest, Sztoczek 2., Hungary, ficzere@kge.bme.hu
}

\begin{abstract}
Even if it sound a bit incredible rapid prototyping (RPT) as production method has been used for decades in other professions. Nevertheless medical science just started discover the possibilities of this technology and use the offered benefits of 3D printing. In this paper authors have investigated the pharmaceutical usage of rapid prototyping.
\end{abstract}

Key words - Rapid Prototyping, 3D printing methods, 3D printed medicines

\section{General requirements}

What would happened if the dreamed product could be hold in hand on the next day, and the appropriate tests can be made? This can be achieved by simultan engineering with RPT, what can be explained as follows: After the idea was born and the drafts have made, the plans created by a computer need to be sent to the right machine what will give a shape to it, and it can be hold in our hand in hours or days after, depending on the complexity of the product (FICZERE ET. AL, 2011). 3D printing is the most common phrase what we can hear, but it is needed to be emphasize that it is just one of the several 3D methods (HORVATH ET. AL., 2014).

Imagine a plastic sculpture. For example, a horse. There are three possible ways to create the horse.

The statue can be prepared along the traditional way, when the horse carve out from a larger piece of plastic block. In this case a subtractive method is used; the plastic block eroded constantly whose size decreasing so finally the desired shape will be formed.

The second way is the shaping of the material. In this case we start with a predetermined size (volume) of the material, then with the help of various shaping forces the desired shape can be created. Forging can be a solution for this when we work with metal, or die-casting in this typical situation. Using this technique it is inevitable that the tools, molds need to be prepared previously with the earlier mentioned, wasteful subtractive method.

Notwithstanding the above, third option is the additive process. Similarly to LEGO ${ }^{\circ}$, where many small building blocks are put together to create something big, the horse is being built up from small elements row by row.

As the first step in the manufacturing process the three-dimensional body need to be planned on a computer. Depending on the complexity of the body what we want to print out another, so called supporting material could be needed. This can be useful when a hollow form is printed, so within certain layers the presence of the material is unnecessary, however it can serve as a basis for the next layer until it solidifies. (FICZERE ET. $A L ., 2013$ ) 


\section{Opportunities for medical use}

Medication with fast prototype production could provide many new opportunities both in the field of medical technologies and self-tailored drug therapies. It is a matter of course that not all of the methods are applicable in health care because of the extraordinary strict medical laws regulating the hygenic, working and medical conditons of the machines producing medications or contacting with medications during the working process; e.g. the regulation of stability, precision, robostness, freedom from distortion, reproducibility etc. To ensure that all of the medications contain the same amount of active substance (substance- uniformity) and be in the same weight (weight-uniformity) between the error borders is also crucial (STASIAK-BETLEJEWSKA, 2015).

The equipments and methods have to be validated and permitted and that procedure could take a long time. They have to achieve the GMP, GRP, GPvP guidelines; so reaching the medical compatiblity of the above listed methods is a significant part of the development. It goes without saying, while a medication seems to have a relatively simple shape without to many contents, it still forms a complex system. Its complexitiy comes from the interactions with the human body which is unpredictable in several cases and takes effect absolutely individually. An absence or presence of a key enzim could lead to different ways of transformation, utilizations and cause severe illnesses or potentially deadly complications.

After all, it should not be noticed that besides the used procedures and machines, the cleanness, trustworthyness and inoffensivity of the used substances are also essential in the pharmacology. The system of active and additive compounds have to fit to the highest standards examining the stability, release, disintegration, features of solution, viscosity, balance and exemption from the chemical and biological pollutants.

The system has to possess appropriate unloose profile and to correspond to all of mentioned criteria. While public awareness just starts infiltrate, printing organic materials has been experimenting for several years and now it's got quite promising results. Probably one of the fastest growing area is food printing, and we might have to begin our journey toward more complex organic system here. Food printing in the Netherlands has already reached a very high standard, and comprehensive experiment have been carried out. The country's interest in food printing is understandable considering the small land at their disposal on agriculture and livestock farming.

Printing a food sounds weird at the first time, but the results of some opinion polls tell us something else. It reveal that the $63 \%$ of people had no objections to the „in vitro" produced meat, and only $25 \%$ of them are strongly against to buy it in the nearby supermarket (MARK, 2015). Of course it is not possible yet, considering that the price of meat patties made like this would be about 3000 USD / piece (without the additional costs). However, if we can rise above our bad feelings, we can realize the importance of these kind of foods. Mostly FDM (Fused Deposition Modelling) machines, or a kind of variation are used to print food. The commodity used as powder or liquid. These devices can not bake or cook yet of course, so in most cases post-production like cooking, baking required. A certain group of sweets can be an exception to this, where the generated heat by the head unit is enough to finalize the food.

According to the consistency of commodity it can be easily foreseeable, that meals having such a form can be transportable more easily, which can save space and money for us, and thanks to lyophilization (when we reversibly dehydrating the raw material) shelf life can be longer. For example these facts could be important when we think of food donations, that needed to delivered to hard to reach and famine-stricken areas of the world.

Another equally important aspect is that livestock can be much more economical, because instead of recovering $30-40 \%$ of the invested energy by slaughtering the cattle to produce meat, with taking out some stemcells we can provide food for a half village without killing the cattle.

Also an invaluable feature of these kinds of foods is that we may be able to change the ingredients. A chicken wing can be printed so that its flavor, shape energy contet is exactly the same as the conventional one, but fat, intolerance and allergy-causing ingredients can be extracted from it. Our food can be prepared folloing our current diet and it is not needed to miss the tasty morsels. We can prevent many diseases, reduce obesity, the risk of cardiovascular diseases or even malnutrition. 


\section{Capsule printing}

It is not surprising then, that the pharmaceutical industry is eagerly towards this technology. We are so close to print out medicines with 3D technology, that FDA (U.S. Food and Drug Administration) authorized the first ever printed medicine in the world this year created by Aprecia Pharmaceuticals Company, which contains levetiracetam against epilepsy! (GOYANES ET. AL., 2015) Although making pills by pressing or by casting is much more faster, it is unquestionable fact that reset the production line during the formulation of a small amount of new drugs takes too much time comparing to 3D printers, where it is not necessary to stop the production of profitably sell products while testing the new active ingredients. The resulting drugs, capsules, can be tested for dissolution, disintegration, friability, strength, reveal the behavior of the new materials simulating human enviroment. (CHEOW, ET AL. 2015)

Such a capsule is showned on the following picture (Fig. 1), on which a new type of releasing system can be seen, appeared as perforation.

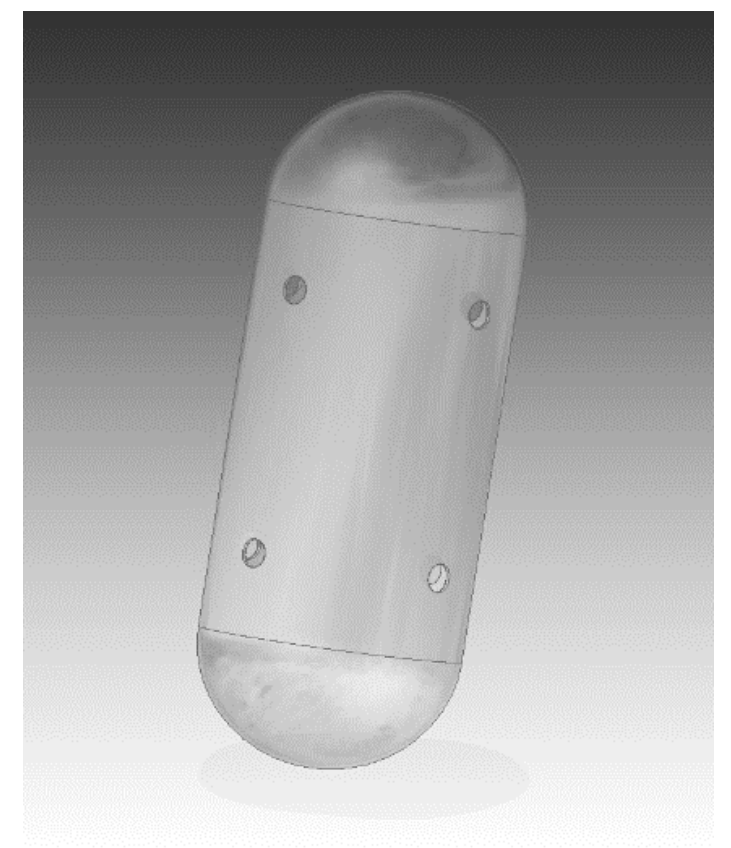

Fig 1.3D printed capsule plan with drug releasing holes source: own model

As it seems clearly on the picture, there are no seams; it is a single-layered capsule, not a matching of two capsule halves as a traditional one. Dissolution is one of the areas where the advantage of this capsule comes out, since where the two halves are aligned with each other the gelatin dissolves more slowly that at the ends of the capsule. Therefore the substance in the capsule start to be wetet unsimultaneously. Drug releasing and duration of pills and capsules created by $3 \mathrm{D}$ printing technology will become more predictable, which makes application more precisly, especially when the patient is taking multiple interacting medicines.

Bridge the problem of interaction, the following solution was planed by me: The concept is to build a capsule from small components like playing with LEGO ${ }^{\circledR}$. Using this principle we have the chance to make drug therapy more unique to the patient, and reduce the amount of the simultaneously used medicines. Components could be combined randomly, picking the right elements by the pharmacist.

Due to the new technology, many unaccomplishable forms can be created, what can elicit a better, faster or even a longer effect, that was not achievable because of the absence of the appropriate technology.

Making unique medicines in public traffic could be very determinative, while in large-scale production it can be an essential step to accelerate the process.

\section{Conclusions}

According to our researches, to technology and the used materials in each technique, Objet - Polyjet, CLIP / DLP, SLA and SLS could be the ways what need to be developed by pharmaceutical indastry. The above mentioned machines could apply those substances what are used in pharmacy, so my researches bring mainly these proceedings into the spotlight.

We belive that this way of development. It is very useful and necessary for the industry, and worth the invested energy, since the siad methods are still unexploited and pharmaceutical industry are absolutly compatible with these innovations. During the process controlled by a computer such things as the reproducibility of weight uniformity or drug content can be more accurate and easier. Because pharmaceutics has the responsibility to develop itself as well, it is highly recommended for workers in this profession to be aware of the new technological methods and see the hidden potentials for progress. Working in a pharmacy doesn't mean 
an excpetion of this. In pharmaceutical industry - although the present circumstances don't allow this - the opportunity to make contact with the patients, control / inspect their medication treatments and make it more personal by warning them to the possible side-effects or replacement options own by those colleagues who works in a pharmacy. In this customization can help us a 3D printer, on which we can set what ingredients should the pill / capsule contains in what quantity, or the shape (size, form) of delivery system what is needed, and then we can easily print out the medicine - for example behind the tare. A so unique medicine that contains the right amount of drugs according to the prescription can replace several other products ensuring the dosage and meaning the ideal medicine for the patients. And don't forget that when these machines find their ways to pharmacies like the so called pharmacy robots, they can save time for the pharmacist who can invest this time into patient care and drug release could be smoother.

\section{Acknowledgement}

Authors are grateful for the support of Erasmus+ LLP Programme.

\section{Literature}

1. Cheow, W. S., KieW, T. Y., Hadinoto, K. (2015): Combining inkjet printing and amorphous nanonization to prepare personalized dosage forms of poorly-soluble drugs. „European Journal of Pharmaceutics and Biopharmaceutics", 96, 314-321. doi:10.1016/j.ejpb.2015.08.012

2. FICZERE P., BORBÁs L., TÖRÖK Á. (2011): Resource analysis of rapid prototyping, „Production Improvement". Stanislaw Borkowski, Manuela Konstanciak, Manuela Konstanciak Trnava: Tripsoft, 2011. pp. 160-172. (ISBN:978-8089291-45-8)

3. FiCZERE P., BORBÁs L., TÖRÖK Á. (2013): Economical investigation of rapid prototyping, „International Journal For Traffic And Transport Engineering” 3:(3) pp. 344-350.

4. Goyanes, A., Martinez, P. R., Buanz, A., Basit, A. W., GAISFORD, S. (2015): Effect of geometry on drug release from $3 D$ printed tablets. „International journal of pharmaceutics”. 494(2):657-663, doi:10.1016/j.ijpharm.2015.04.069

5. HORVÁTH E., TÖRÖK Á., FICZERE P., ZÁdOR I., RÁCZ P. (2014): Optimisation of Computer-aided Screen Printing Design, „Acta Polytechnica Hungarica” 11:(8) pp. 29-44.

6. MARK J. POST (2015): Medical Technology to produce Beef, Department of Physiology Maastricht University, The Netherlands. Venlo

7. MURPHY S. V., ATALA A. (2014): 3D bioprinting of tissues and organs, ,Nature Biotechnology” 32, 773 785 (2014), doi:10.1038/nbt.2958

8. StASIAK-BetLEJEWSKA R. (2015): Value Engineering Application in the American Transportation Industry, „Period. Polytech. Transp. Eng.,” (43)4, 206-210, DOI: 10.3311/PPtr.8448 